\title{
Analytical Modeling of an Axial Field Magnetic Coupler with Cylindrical Magnets
}

\author{
L. Belguerras, S. Mezani, T. Lubin \\ Université de Lorraine, GREEN, F-54000 Nancy, France
}

\begin{abstract}
A 2D analytical model is proposed to calculate the magnetic field distribution in axial magnetic coupler with disc magnets. The 2D simplification is possible by assuming an infinite radius of the ferromagnetic yokes. Then, an analytical solution is obtained for a single magnet. This solution allows the determination of the magnetic field due to all the magnets so the total magnetic field is obtained by superposition. To demonstrate the validity of the model, the analytical results are compared with those obtained from 3-D FE simulations and from experimental tests carried out on a magnetic coupler equipped with disc magnets and HTS pancake coils.
\end{abstract}

Index Terms-Analytical model, Disc magnet, Finite Elements, HTS pancake coil, Magnetic coupler

\section{INTRODUCTION}

$\mathrm{M}$ AGNETIC Couplings (or couplers) (MCs) are used to transmit a torque from a prime mover to its load without contact [1]. Compared to their mechanical counterparts, MCs have several advantages such as low mechanical vibrations, less wear, natural protection against overloads and permit misalignment. Furthermore, they are widely used in industrial applications which need a hermetic isolation between the drive and the load sides by means of a separation wall which can be air, vacuum, fluid or other media. They are used in magnetic drive pumps and magnetic mixers for seal-less applications (i.e. nuclear, chemical, pharmaceutical and food industries).

Radial and axial field MCs topologies exist; they both consist of two rare earth PM rotors owing to their high torque transmission capabilities. The design of the axial field topology requires 3D computations.

The finite-element (FE) method is accurate as it considers the nonlinearity of magnetic materials and the actual coupler geometry [2]. However, FE methods require long computation time which make them unsuitable for optimization purposes which require many repetitive computations.

Reluctance network can also be used to compute magnetic couplings and present less computational effort compared to FE analyses [3].

Biot-Savart like formulas are used to determine the magnetic field distribution in ironless structures (magnets in free space). Combined to the method of images, iron walls can be accounted for although the cpu time increases [4]-[5].

Closed form solution of the magnetic field can be derived for sector PMs coupler. This requires a resolution in Cartesian coordinates so a linearized geometry is considered [6]. The magnetic field is then calculated in 3D by solving the PDE using separation of variables. A torque formula is also derived. This linearization is no more possible for disc PMs couplers so

Manuscript received May 8, 2020. Corresponding author: S. Mezani (email: smail.mezani@univ-lorraine.fr).

Digital Object Identifier (inserted by IEEE) a 3D analytical solution cannot be obtained. We propose in this paper a $2 \mathrm{D}$ analytical model for the determination of the magnetic field distribution in disc magnets MCs.

\section{Studied Magnetic CouPleR}

The magnetic coupler considered in this study is an axial field one which consists of two cylindrical rotors facing each other and separated by an airgap, Fig. 1 .

The first rotor is composed of axially magnetized cylindrical PMs mounted on the surface of a ferromagnetic yoke. Each magnet has an opposite polarity compared to its neighbouring ones to create a spatially variable magnetic field in the airgap.

The second rotor is composed of pancake coils mounted on a ferromagnetic yoke. These coils are made from High Temperature Superconducting (HTS) wires.

The main parameters of the coupler [7] are given in Table I. The HTS coils can be supplied by dc current values up to $25 \mathrm{~A}$ which result in a high current density in excess of $30 \mathrm{~A} / \mathrm{mm}^{2}$ at a cooling temperature of $77 \mathrm{~K}$ (liquid nitrogen). This coupler has been constructed and successfully tested in [7]. Airgap magnetic fields and torque measurements will serve to validate the analytical model developed here for this coupler.

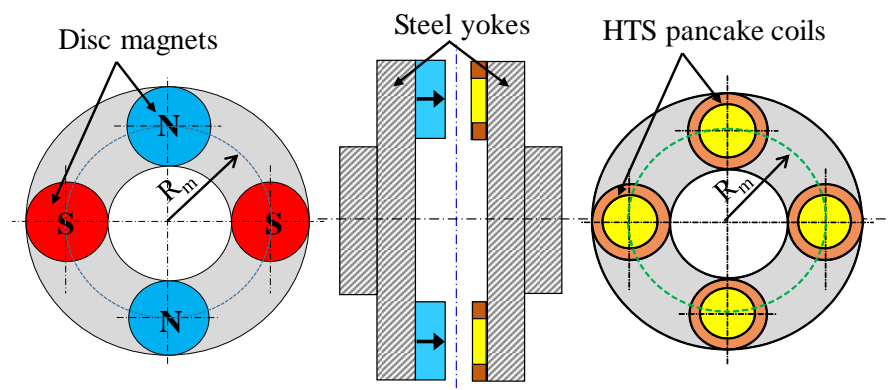

Fig. 1. Geometry of the studied axial field magnetic coupler $(\mathrm{p}=2)$ 
TABLE I

MAIN PARAMETERS OF THE STUDIED HTS MAGNETIC COUPLER

\begin{tabular}{lll}
\hline \hline Symbol & \multicolumn{1}{c}{ Description } & \multicolumn{1}{c}{ Value } \\
\hline$R$ & PM radius & $50 \mathrm{~mm}$ \\
$Z_{l}$ & PM thickness & $10 \mathrm{~mm}$ \\
$R_{c i}$ & HTS coil inner radius & $25 \mathrm{~mm}$ \\
$R_{c o}$ & HTS coil outer radius & $50 \mathrm{~mm}$ \\
$Z_{c}$ & HTS coil thickness & $3.5 \mathrm{~mm}$ \\
$Z_{e}$ & Air-gap & Variable $(9-20 \mathrm{~mm})$ \\
$R_{m}$ & Coupler mean radius & $85 \mathrm{~mm}$ \\
$p$ & Number of pole pairs & 2 \\
$B_{\text {rem }}$ & PM remanence (NdFeB) & $1.25 \mathrm{~T}$ \\
$N$ & Number of turns/coil & 63 \\
$I_{\text {coil }}$ & HTS coil current & Variable $(0-25 \mathrm{~A})$ \\
\hline
\end{tabular}

\section{Method OF ANALYSIS}

We aim in this paper to compute the torque produced by the device. This torque can be computed using the Laplace force exerted on the pancake coils. We need then to determine the magnetic field due to the magnets only.

The geometry and the nature of the magnetic field distribution in the studied coupler requires 3D computations. However, under some reasonable assumptions, it is possible to simplify the analysis and use a 2D model to obtain closedform expressions for the magnetic field distribution due to the PMs. Assuming the linearity of the ferromagnetic materials, the magnetic field created by the $2 p$ magnets is the sum of the fields due to each magnet. If the yokes are assumed to have an infinite radius, it is then possible to calculate the magnetic field distribution of a single magnet in 2D.

\section{A. Magnetic field due to a single PM}

We propose to compute analytically the magnetic field generated by a single disc PM. For simplicity, we assume that the iron yokes have an infinite magnetic permeability and the magnet has a unity relative permeability.

An axisymmetric 2D model in a cylindrical coordinate system $(r, z)$ is considered. As shown in Fig.2, the whole domain is divided into two regions: the PM region (I) and the air region (II). The magnetization distribution in region $\mathrm{I}$ is also provided.

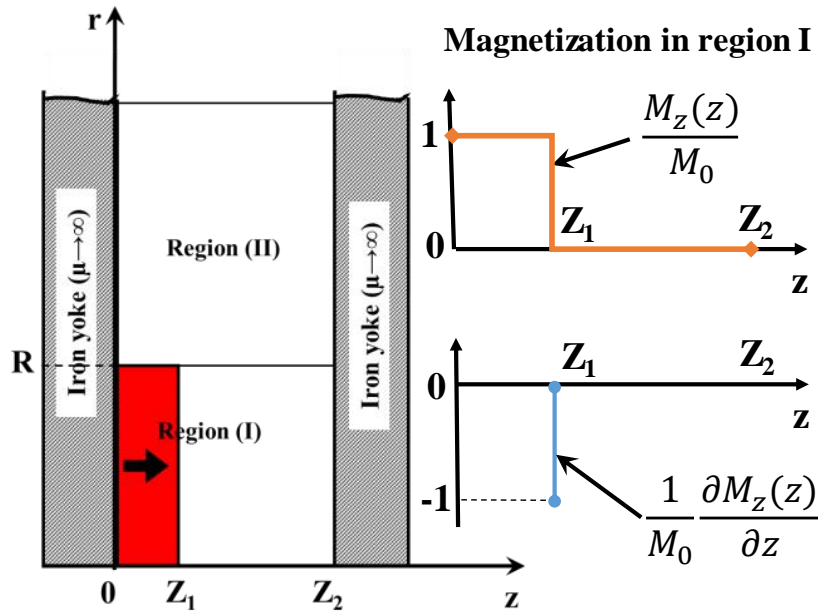

Fig. 2. Definition of sub-domains and magnetization distribution
A magnetic scalar potential formulation is used to solve the problem. The equations to solve in regions I and II are given by (1) and (2), respectively.

$\nabla^{2} \varphi_{I}=\partial M_{z} / \partial z$

$\nabla^{2} \varphi_{I I}=0$

As we can see in Fig. 2, the source term on the rhs of (1) can be written as

$\partial M_{z} / \partial z=-M_{0} \delta\left(z-z_{1}\right)$

In (3), $\delta\left(z-z_{1}\right)$ is the Kronecker delta function and $M_{0}=B_{r e m} / \mu_{0}$ where $B_{r e m}$ represents the remanent flux density of the PM.

The method of separation of variables is used to solve (1) and (2). We use the boundary conditions on $\mathrm{z}=0$ and $\mathrm{z}=\mathrm{Z}_{2}$ to determine the eigenvalues and eigenfunctions of the homogeneous problem (Laplace equation). These boundary conditions correspond to a nil tangential magnetic field, viz.

$\left.\frac{\partial \varphi_{I, I I}}{\partial r}\right|_{z=0, Z_{2}}=0$.

The obtained eigenvalues are noted $\beta_{n}=\omega_{n}^{2}$ such as

$\omega_{n}=n \pi / Z_{2} \quad n=1,2,3 \ldots$

The corresponding eigenfunctions are $f_{n}(z)=\sin \left(\omega_{n} z\right)$

The differential equation with respect to $r$ is a modified Bessel equation whose solution is the superposition of the 0 order modified Bessel functions of the first kind $I_{0}$ and the second kind $K_{0}$.

In order to get a finite solution on the boundary $r=0$, the general solution of Poison's equation in region I is

$\varphi_{I}(r, z)=\sum_{n=1}^{\infty}\left[A_{I n} I_{0}\left(\omega_{n} r\right)+\alpha_{n}\right] \sin \left(\omega_{n} z\right)$

with $\alpha_{n}=\frac{2 M_{0}}{z_{2} \omega_{n}^{2}} \sin \left(\omega_{n} z_{1}\right)$

The term $\alpha_{n} \sin \left(\omega_{n} z\right)$ represents the particular solution of (1) obtained by the projection of (3) on the eigenfunctions basis $f_{n}(z)$.

In order to get a finite solution on the boundary $r \rightarrow \infty$, the general solution of Laplace's equation in region II is

$\varphi_{I I}(r, z)=\sum_{n=1}^{\infty} A_{I I n} K_{0}\left(\omega_{n} r\right) \sin \left(\omega_{n} z\right)$

In (5) and (6), the constants $A_{I n}$ and $A_{I n}$ are determined using the continuity of the tangential magnetic field and the normal flux density at $r=R$ 


$$
\begin{aligned}
& A_{I n}=\frac{-\alpha_{n} K_{1}\left(\omega_{n} R\right)}{I_{0}\left(\omega_{n} R\right) K_{1}\left(\omega_{n} R\right)+I_{1}\left(\omega_{n} R\right) K_{0}\left(\omega_{n} R\right)} \\
& A_{I n}=\frac{\alpha_{n} I_{1}\left(\omega_{n} R\right)}{I_{0}\left(\omega_{n} R\right) K_{1}\left(\omega_{n} R\right)+I_{1}\left(\omega_{n} R\right) K_{0}\left(\omega_{n} R\right)}
\end{aligned}
$$

where $I_{1}$ and $K_{1}$ are the modified Bessel functions, of order 1, of the first and second kind, respectively.

From the magnetic scalar potential, it is easy to derive closed form solutions of the magnetic field strength $\overrightarrow{H_{I, I I}}=-\nabla \varphi_{I, I I}$ and the flux density using the constitutive law of the different media. For example, the axial flux density expression in air regions is given by

$$
\left\{\begin{array}{l}
B_{z I}(r, z)=\sum_{n=1}^{\infty}-\omega_{n} A_{I n} I_{0}\left(\omega_{n} r\right) \cos \left(\omega_{n} z\right) \quad \mathrm{r} \leq \mathrm{R}, \mathrm{Z}_{1} \leq z \leq \mathrm{Z}_{2} \\
B_{z I I}(r, z)=\sum_{n=1}^{\infty}-\omega_{n} A_{I I n} K_{0}\left(\omega_{n} r\right) \cos \left(\omega_{n} z\right) \mathrm{r} \geq \mathrm{R}, \mathrm{Z}_{1} \leq z \leq \mathrm{Z}_{2}
\end{array}\right.
$$

To check the validity of the proposed model, Fig. 3 compares flux density distributions computed analytically and numerically using 2D finite elements [8]. The geometrical and physical parameters of the magnet are these given in Table I while the airgap is set to $Z_{\mathrm{e}}=20 \mathrm{~mm}$. The studied domain axial length as defined in Fig. 1. is then $Z_{2}=Z_{1}+Z_{e}+Z_{c}=33.5 \mathrm{~mm}$.

The curves are plotted along a line in the $\mathrm{z}$ direction (from $\mathrm{z}=0$ to $\mathrm{z}=\mathrm{Z}_{2}$ ) located at a radius $\mathrm{r}=25 \mathrm{~mm}$. A very good agreement is noticed between the FE predictions and the analytical results for both radial and axial flux density distributions.

\section{B. Magnetic field due to the PM rotor}

The PM rotor consists of $2 \mathrm{p}$ disc magnets disposed on the ferromagnetic yoke as shown in Fig. 1.

To each magnet $\mathrm{k}(\mathrm{k}=1,2, \ldots, 2 \mathrm{p})$, let us define a local coordinate system $\left(\mathrm{x}_{\mathrm{k}}, \mathrm{y}_{\mathrm{k}}, \mathrm{z}\right)$ such as $r_{k}=\sqrt{x_{k}^{2}+y_{k}^{2}}$ (same $\mathrm{z}$ coordinate for all these coordinate systems). We also define a global coordinate system $(\mathrm{x}, \mathrm{y}, \mathrm{z})$ such as

$$
\left\{\begin{array}{l}
x=x_{k}+x_{0 k} \\
y=y_{k}+y_{0 k}
\end{array}\right.
$$

If the global coordinate system is attached to the center of the coupler and the first magnet is centered on the $\mathrm{x}$ axis at a distance $R_{m}$, it is easy to show that

$$
\left\{\begin{array}{l}
x_{0 k}=R_{m} \cos ((k-1) \pi / p) \\
y_{0 k}=R_{m} \sin ((k-1) \pi / p)
\end{array}\right.
$$

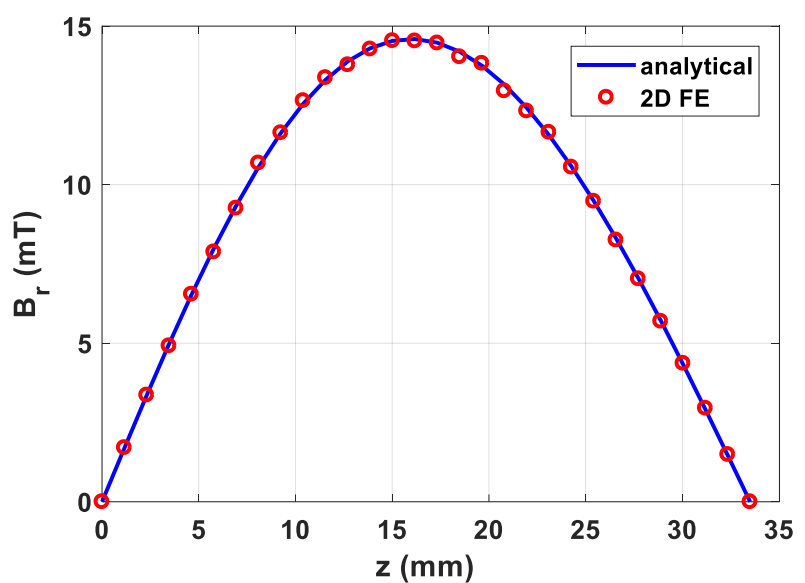

(a)

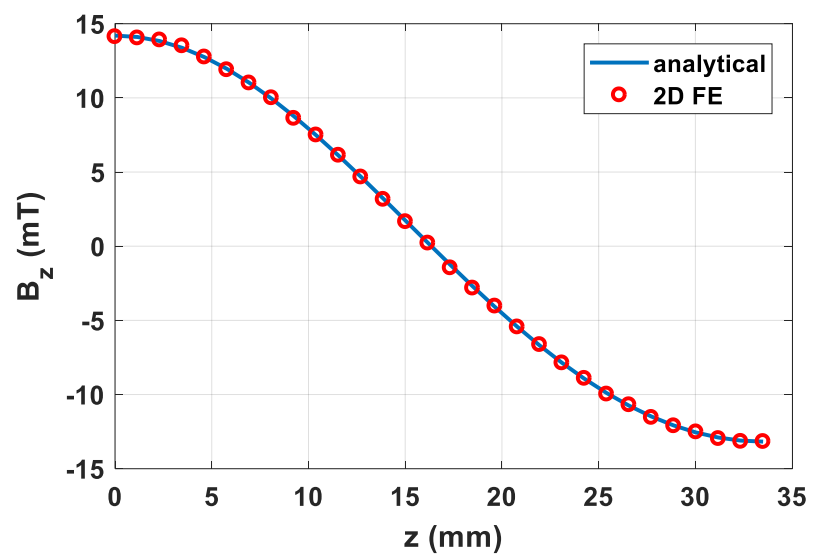

(b)

Fig. 3. Radial (a) and axial (b) flux densities vs. $\mathrm{z}$ at $\mathrm{r}=25 \mathrm{~mm}\left(\mathrm{Z}_{\mathrm{e}}=20 \mathrm{~mm}\right)$

For example, the axial flux density created by the $2 p$ magnets on the point $\mathrm{P}(\mathrm{x}, \mathrm{y}, \mathrm{z})$ with $\mathrm{Z}_{1} \leq z \leq \mathrm{Z}_{2}$ is

$B_{z}(x, y, z)= \begin{cases}\sum_{k=1}^{2 p}(-1)^{k-1} \sum_{n=1}^{\infty} \omega_{n} A_{I n} I_{0}\left(\omega_{n} r_{k}\right) \cos \left(\omega_{n} z\right) & \mathrm{r}_{k} \leq \mathrm{R} \\ \sum_{k=1}^{2 p}(-1)^{k-1} \sum_{n=1}^{\infty} \omega_{n} A_{I I n} K_{0}\left(\omega_{n} r_{k}\right) \cos \left(\omega_{n} z\right) & \mathrm{r}_{k} \geq \mathrm{R}\end{cases}$

In Fig. 4, the analytical results are compared with those obtained using 3D FE computations. The computed waveforms of the axial flux density distribution at $\mathrm{z}=20 \mathrm{~mm}$ along a circle of radius $\mathrm{R}_{\mathrm{m}}$ (Fig.4.a) and along a radial line shifted by $30^{\circ}$ with respect to the $\mathrm{x}$ axis (Fig.4.b) are in close agreement.

The analytical computations of the axial flux density have been also compared to measured ones carried out on a rectangular surface Sg shown in Fig. 5. The measured values. and the analytical results are given in Fig. 6. The agreement is pretty good as the relative difference does not exceed $10 \%$. 


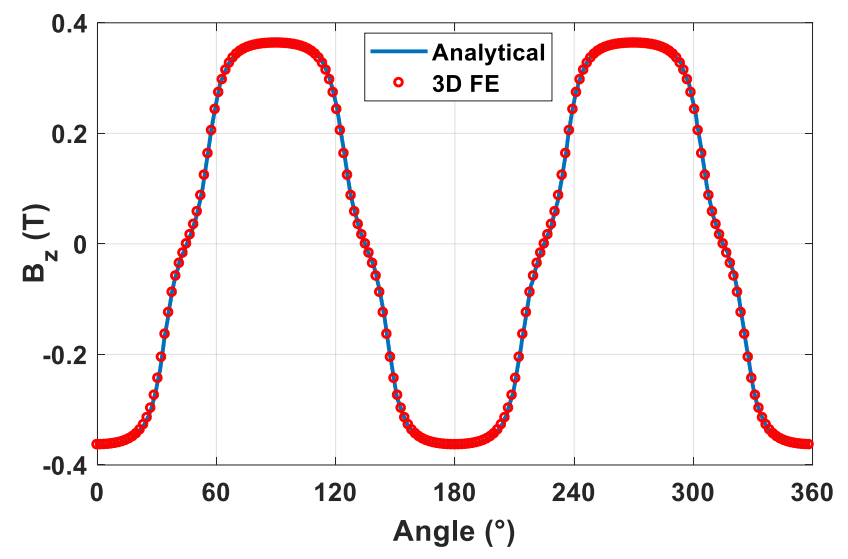

(a)

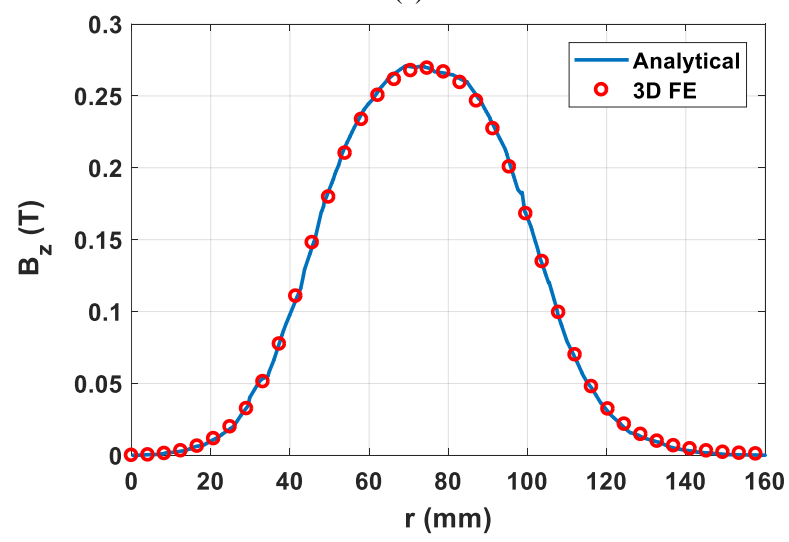

(b)

Fig. 4. Axial flux density distribution in the airgap at $\mathrm{z}=20 \mathrm{~mm}$ (a) along a circle of radius $R_{m}$ (b) along a radial line

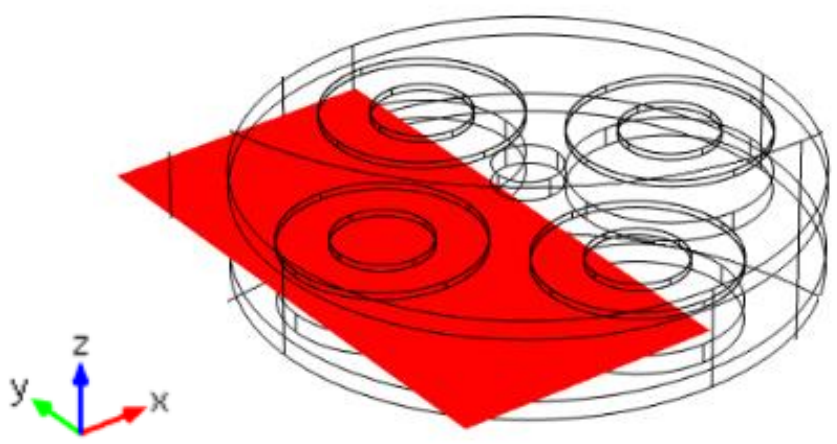

Fig. 5. Definition of the surface $\mathrm{Sg}$ in the air gap $(\mathrm{z}=20 \mathrm{~mm})$

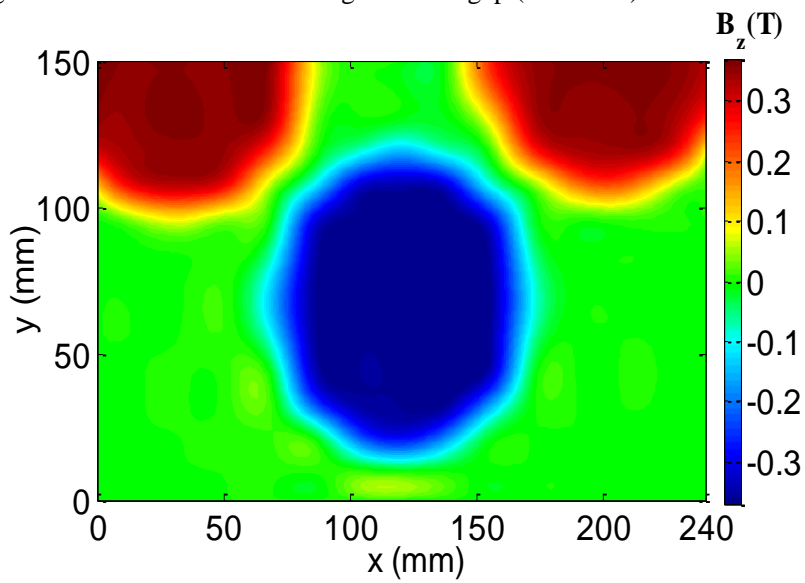

(a)

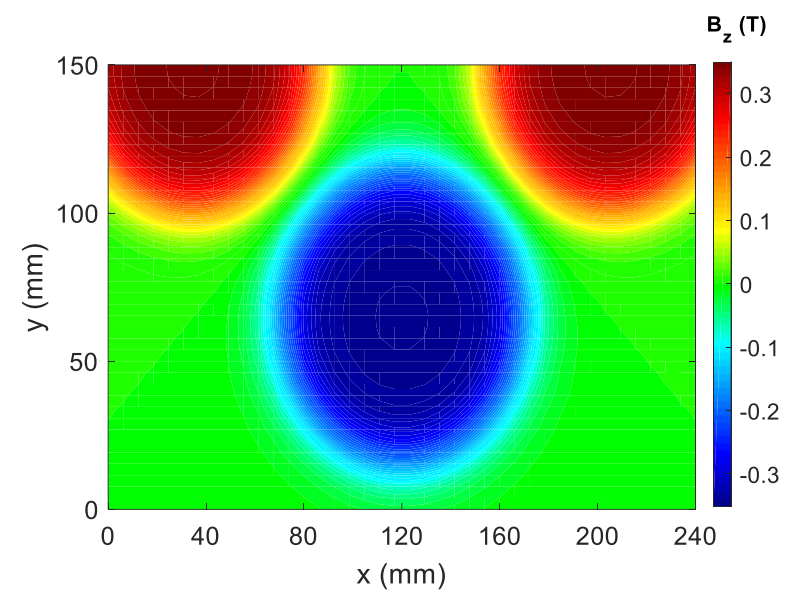

(b)

Fig. 6. Distribution of the axial flux density $\mathrm{B}_{z}$ over the surface $\mathrm{Sg}$ (a) measured (b) analytical

\section{Electromagnetic torque}

The torque is computed via the Laplace force exerted on the HTS coils. The force density $\vec{f}$, in $\mathrm{N} / \mathrm{m}^{3}$, and the torque density $\vec{c}$, in $\mathrm{Nm} / \mathrm{m}^{3}$, are deduced from the following cross products

$\left\{\begin{array}{l}\vec{f}=\vec{J} \times \vec{B} \\ \vec{c}=\vec{r} \times \vec{f}\end{array}\right.$

Where $\vec{B}$ is the flux density due to the PM rotor, $\vec{J}$ the current density in the HTS coil and $\vec{r}$ the lever arm vector. According to the notations of Table I, the amplitude of $\vec{J}$ is calculated by $J=N I_{\text {coil }} /\left(Z_{c}\left(R_{c o}-R_{c i}\right)\right)$.

To compute the torque value, we need to integrate (13) over the $2 \mathrm{p}$ coils volume. However, because of the geometric and magnetic field distribution periodicities, we just need to integrate over one coil and multiply the result by $2 p$ to get the total torque.

To perform the integration, let us define a coordinate system $\left(\mathrm{x}_{\mathrm{c}}, \mathrm{y}_{\mathrm{c}}, \mathrm{z}\right)$ attached to the center of one coil. These coordinates are related to the global coordinates system by

$\left\{\begin{array}{l}x=x_{c}+x_{0 c} \\ y=y_{c}+y_{0 c}\end{array}\right.$

The angle between $(\mathrm{x}, \mathrm{y}, \mathrm{z})$ and $\left(\mathrm{x}_{\mathrm{c}}, \mathrm{y}_{\mathrm{c}}, \mathrm{z}\right)$ frames is noted $\theta$, so

$\left\{\begin{array}{l}x_{0 c}=R_{m} \cos (\theta) \\ y_{0 c}=R_{m} \sin (\theta)\end{array}\right.$

Hence, the coil's coordinates $\left(\mathrm{x}_{\mathrm{c}}, \mathrm{y}_{\mathrm{c}}, \mathrm{z}\right)$ and the magnet coordinates $\left(\mathrm{x}_{\mathrm{k}}, \mathrm{y}_{\mathrm{k}}, \mathrm{z}\right)$ are linked by 
$\left\{\begin{array}{l}x_{k}=x_{c}+x_{0 c}-x_{0 k} \\ y_{k}=y_{c}+y_{0 c}-y_{0 k}\end{array}\right.$

We can now express all the vector quantities in (13) with respect to the coordinate system $\left(\mathrm{x}_{\mathrm{c}}, \mathrm{y}_{\mathrm{c}}, \mathrm{z}\right)$. The torque is computed by integration over the coil's volume $V_{c}=S_{c} Z_{c}$ where $S_{c}=\pi\left(R_{c o}^{2}-R_{c i}^{2}\right)$ is the cross section area of the coil. Since the integration with respect to $\mathrm{z}$ is easy, we only need to perform a double integral over the cross section area of the coil. Unfortunately, this integration is not feasible in an analytical way so a numerical integration is necessary to get the torque value.

Figure 7 presents the measured and the computed static torque vs. load angle curves for a current $\mathrm{I}_{\text {coil }}=25 \mathrm{~A}$ and for 2 airgap values $\left(Z_{\mathrm{e}}=9 \mathrm{~mm}\right.$ and $\left.Z_{\mathrm{e}}=20 \mathrm{~mm}\right)$. It can be seen that the analytical and the $3 \mathrm{D}$ FE predictions are very close. The computed pull-out torque for $Z_{\mathrm{e}}=9 \mathrm{~mm}$ is about $28 \mathrm{Nm}$ while the measurements give $33 \mathrm{Nm}$ (a relative difference of about 9.7\%). For the airgap of $20 \mathrm{~mm}$, the measured and calculated pull-out torques are $19.6 \mathrm{Nm}$ and $16.3 \mathrm{Nm}$, respectively (16\% difference). These discrepancies between the computed and the measured torques may result from the difficulties to perform the tests in a cryogenic environment [7]:

- Uncertainties in the measured airgap thickness

- Static friction torque due to the "pulley-rope" system and to the angular-contact ball bearing used in the PM rotor. Of course, this friction torque increases with the very low temperature of the PMs rotor which is very close to the HTS rotor immerged in liquid nitrogen (weak thermal insulation through the airgap).

The computation time for a single position is about $10 \mathrm{~s}$ with the 3D FE method in which one pole with antiperiodic boundary conditions is considered. The proposed model requires $0.8 \mathrm{~s}$. It is then a useful tool for the design and optimization of the studied coupler.

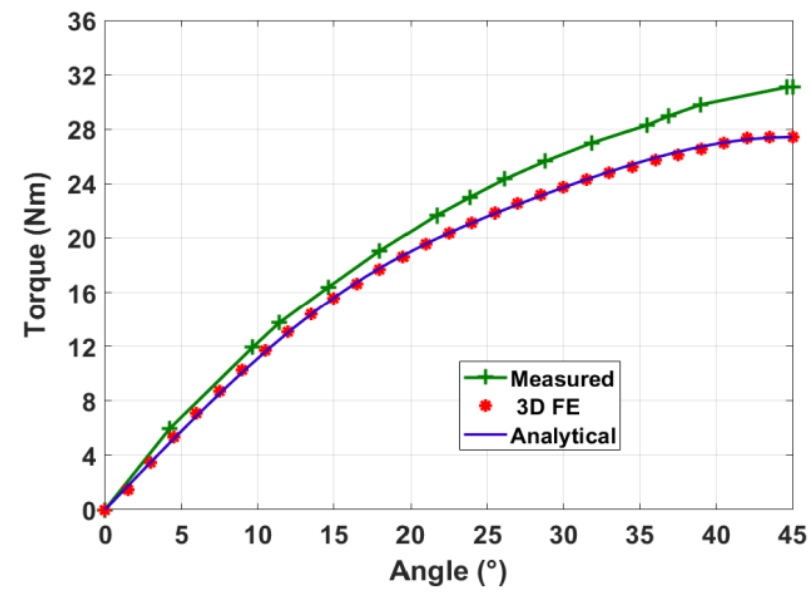

(a)

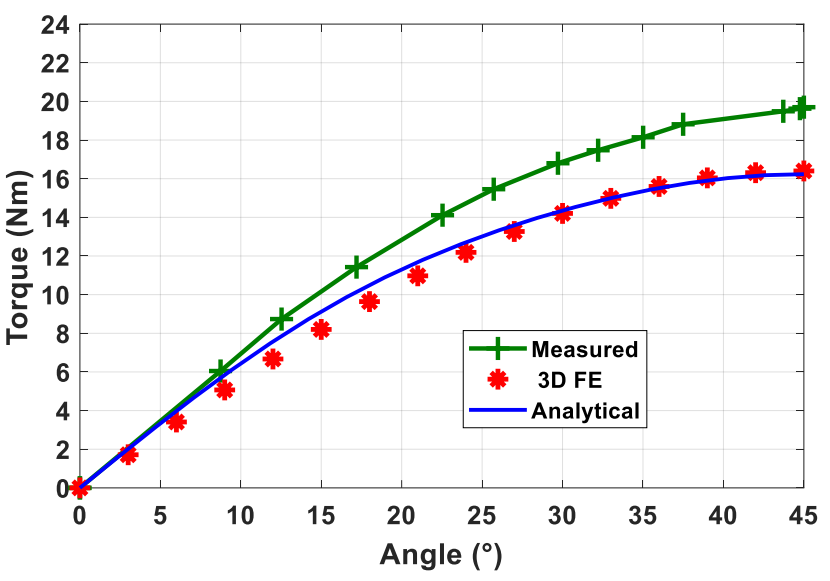

(b)

Fig. 7. Static torque vs. load angle ( $I_{\text {coil }}=25$ A) (a) $Z_{\mathrm{e}}=9 \mathrm{~mm}$ (b) $Z_{\mathrm{e}}=20 \mathrm{~mm}$.

\section{CONCLUSION}

An analytical model is proposed to calculate the magnetic field distribution in a magnetic coupler equipped with disc magnets and HTS pancake coils. The model is validated through experimental results as well as 2-D and 3-D FE computations of the magnetic field distribution and the torque. We have shown that the proposed model is very accurate and computationally very efficient. In the future, the model will be adapted to calculate the magnetic field due to the pancake coil. This will allow a more realistic design of the coupler by including a calculation of the operating current of the HTS coils.

\section{REFERENCES}

[1] M. Cong, T. Li, "Design and Application of Magnetic Coupling Used for Ultra-high Vacuum Robot", 15th International conference on Mechatronics and Machine Vision in Practice (M2VIP 08), pp.346-351, 2-4 Dec 2008, Auckland, New-Zealand.

[2] W. Y. Lin, L. P. Kuan, W. Jun, and D. Han, "Near-optimal design and 3D finite element analysis of multiple sets of radial magnetic couplings," IEEE Trans. Magn., vol. 44, no. 12, pp. 4747-4753, Dec. 2013.

[3] C. Xikang, L.Wei, Z. Yang, L. Sitong, and L. Weiqi, "A Concise Transmitted Torque Calculation Method for Pre-Design of Axial Permanent Magnetic Coupler," IEEE Trans. on Ener. Conv., vol. 35, no. 2, pp. 938-947, Jun. 2020.

[4] J. P. Yonnet, S. Hemmerlin, E. Rulliere, and G. Lemarquand, "Analytical calculation of permanent magnet couplings," IEEE Trans. Magn., vol. 29, no. 6, pp. 2932-2934, Jun. 1993.

[5] R. Ravaud, G. Lemarquand, V. Lemarquand, and C. Depollier, "Permanent magnet couplings: Field and torque three-dimensional expressions based on the Coulombian model," IEEE Trans. Magn., vol. 45, no. 4, pp. 1950-1958, Apr. 2009.

[6] B. Dolisy, T. Lubin, S. Mezani and J. Lévêque, "A New Analytical Torque Formula for Axial Field Permanent Magnets Coupling," IEEE Trans. on Ener. Conv., vol. 30, no. 3, pp. 892-899, Sept. 2015.

[7] L. Belguerras, S. Mezani, T. Lubin, J. Lévêque and A. Rezzoug, "High temperature superconducting axial field magnetic coupler: realization and test, " Supercond. Sci. Technol., 28, 095003 (12pp), 2015.

[8] D. C. Meeker, Finite Element Method Magnetics Version 4.2 (Jan 12 2016 Build) http://www.femm.info. 